\title{
"Struggling to Bridge the Intergeneration Gap": Iranian Parents' approaches to their Adolescent Sons' Sexual Upbringing-a qualitative study
}

\section{zahra Rouhparvar}

Ahvaz Jundishapur University of Medical Sciences: Ahvaz Jondishapour University of Medical Sciences mojgan javadnoori (D mojganjavadnoori@gmail.com )

Ahvaz Jundishapur University of Medical Sciences: Ahvaz Jondishapour University of Medical Sciences https://orcid.org/0000-0001-7645-3629

\section{Shadab Shahali}

Tarbiat Modares University Faculty of Medical Sciences

\section{Research}

Keywords: Sex education, child rearing, Parents, Adolescent, Iran

Posted Date: June 9th, 2021

DOI: https://doi.org/10.21203/rs.3.rs-596710/v1

License: (c) (i) This work is licensed under a Creative Commons Attribution 4.0 International License. Read Full License 


\section{Abstract}

\section{Background}

Children's sexual education has an important role in adolescent sexual health; however sexual upbringing is one of the most challenging dimensions of education for families, especially in Muslim societies experiencing transition to modernity. There is little information about sexuality upbringing for boys in Iran. The present study aimed to explain parents' strategies in the sexual upbringing of adolescent boys in Iran.

\section{Methods}

This qualitative study was conducted in 2017 in Ahvaz, Iran. The qualitative data were collected through a purposeful sampling method, with a maximum variation by conducting semi-structured in-depth interviews and focused group discussions with 27 parents from the middle/high social class having 1019-year-old adolescent sons. Qualitative data were analyzed using conventional qualitative content analysis.

\section{Results}

Parents' strategies for their sons' sexuality upbringing emerged in five categories: control, monitoring, and restriction; abstinence as the main content of sexual upbringing; endeavoring for peace and tolerance; Criticizing the pathologic attitude to sexual instinct; and hope for social learning. The theme "Struggling to bridge the inter-generation gap" emerged from the categories.

\section{Conclusion}

Iranian parents are facing uncertainty regarding sexual education for their adolescent boys. All approaches finally resulted in abstinence. Abstinence is the central concept in their sexuality upbringing style for their sons. so, sexual relationship is a red line issue in sexuality upbringing. Their conservative peaceful approach tries to reduce the intergeneration gap.

\section{Plain English Summary}

Children's sexual education has an important role in adolescent sexual behaviors and health; however sexual upbringing is one of the most challenging dimensions of education in families, especially in Muslim societies experiencing transition to modernity. There is little information about sexuality upbringing for boys in Iran. The present study aimed to explain parents' strategies in the sexual upbringing of adolescent boys in Iran. Results show that Parents' strategies for their sons' sexuality upbringing include: control, monitoring, and restriction; abstinence as the main content of sexual upbringing; endeavoring for peace and tolerance; Criticizing the pathologic attitude to sexual instinct; and hope for social learning. Parents seem to be "Struggling to bridge the inter-generation gap". We concluded 
approaches finally resulted in abstinence. Their conservative peaceful approach tries to reduce the intergeneration gap. Abstinence is the axial concept; so, sexual relationship is the red line issue in sexuality upbringing.

\section{Background}

Sexuality education is recognized as a human right and an indispensable necessity for development, but most adolescents have little access to sexual education, especially in developing countries [1]. However, many adolescents engage in high-risk sexual behaviors or sexual abuse that can lead to morbidity and mortality [2].

Several studies have indicated that parents-adolescents sexual communication is associated with lower risky behaviors in adolescents and the creation of more conservative sexual beliefs and attitudes in adolescents [3]. They have also found the positive impact of school-based sexual education [4], but there is a lot of cultural resistance to sexual education in Asian cultures. Most parents traditionally believe that talking to children about sex can lead to early sexual activity; labeled as sexual deviation or unrestrained sex $[5,6]$. Misunderstanding of nature, purpose, and effects of sexuality upbringing can lead to resistance [7]. In the past decades, a great number of boys reported that they had no significant sexual communication with their parents and received most of their sexual information from friends and the media [8]. However, adolescent boys in the United States have safer sexual behaviors, fewer sex partners, and start sex at an older age due to sexuality education [9].

As a Muslim country, which has experienced a transition to modernity since two decades ago, Iran has faced cultural challenges and major intergenerational conflicts. The sociocultural challenges have been the most important challenges facing adolescents' sexuality education in Iran [6]. Friends in the first place and family in the sixth place have been the sources of sexual information for students in Iran [10]. According to recent evidence, at least $20 \%$ of Iranian adolescents' experience sexual intercourse [11-12], and AIDS transmission through unprotected sex has increased dramatically [13].

Despite numerous studies on adolescents' sexuality education, most of them cover only girls and there are too few studies on boys, especially in Iran. Lack of focus on boys' sexuality may have cultural roots [14]. The qualitative study on parents' experiences and perceptions of sexuality education of boys in Muslim society can help identify basic social processes in this phenomenon. The present study aimed to explain parents' strategies for sexuality education of adolescent boys.

\section{Methods}

The qualitative approach was used with a conventional qualitative content analysis method to explore the Iranian parents' approaches to their adolescent sons' sexual upbringing. The data were collected from January to June 2017. Participants were a total of 27 parents who had adolescent boys aged 10 to 19 years from the middle/high social class living in Ahvaz, the capital of Khuzestan province. They were 
recruited in the study by purposive sampling. Due to the sensitivity of the subject and constraints of the Directorate of Education, the research subject was announced according to principals' consent. Eligible and volunteer parents were recruited. The first researcher contacted the volunteer parents and scheduled for an interview or group discussion. Interviews and focus group discussions were held at schools. In depth semi-structured interviews were conducted with six fathers and five mothers. Two focused group discussions were held separately with eight mothers and eight fathers.

The study objectives and process were explained to the participants. Verbal informed consent was obtained. Interviews were recorded after assuring them of confidentiality and anonymity of information. The interviews began with a general question (please tell me about your experience of your son's puberty and sexuality upbringing) to allow participants to freely express their experience with sexuality upbringing for their sons. Afterward, exploratory terms were used to deepen interviews and access to much richer data based on participants' response, for instance, "Explain more about this case by examples?" Finally, they were asked to contact the researcher if they had something in their mind later. One interview was held for each participant. Data collection was continued until data saturation occurred. The interviews lasted for 30 to 70 minutes and length of the two group discussions were 80 and 100 minutes.

Data collection and data analysis were conducted simultaneously, using proposed methods by Graneheim \& Lundman: 1 . Transcribing all interviews immediately after each interview; 2 . Reading the transcripts for several times to understand its overall content; 3 . Determining meaning units and primary codes; 4. Classifying similar basic codes in more comprehensive classes, and 5. Determining the latent content contained in data [15].

Four factors namely Credibility, Dependability, Confirmability, and Transferability were used according to Lincoln and Guba to ensure the rigor and trustworthiness of data [16]. The participant review method was also used to validate data and codes; in other words, transcripts were returned to participants after coding to ensure the correctness of codes and interpretation; codes, which did not indicate participants' intention, were corrected. Efforts were made to have maximum variation participants in terms of age and education. For the transferability of findings, participants' quotations were objectively presented.

\section{Results}

The parents aged 30 to 53 years, and their educational level varied from high school to master. They have governmental or self-employed jobs.

Eighteen sub-categories, five main categories, and eventually a theme emerged from 73 codes by analyzing parents' experiences and perceptions, and adopted strategies by parents for the sexuality upbringing of adolescent boys (Table 1 ).

Table 1. Parents' strategies for sexuality upbringing of adolescent boys 


\section{Sub-categories}

1. Unobvious monitoring

2. Restricting access to sexual information

3. Controlling and restricting relationships

4. Silence and dodge

5. Avoiding extreme limitations

1. Deviating adolescents' thoughts

from the sexual instinct

2 Trying to inhibit sexual orientations and experiences

3. Resorting to the religion as a lever

4. Learning and warning

1. Avoiding violent behavior

2. Empathy and intimacy with adolescents

3. A hopeful look at puberty changes

1. Criticism of cultural taboos

2. Trying to desensitize the sexual issues

3. Empowerment instead of limitations

1. Self-modeling for adolescents Hope for social learning

2. Metaphorical education

3. Optimism about the adequacy of deterrent boundaries

\section{Categories}

Control, monitoring and

restriction
Endeavoring for peace and tolerance
Criticizing the pathologic attitude to sexual instinct
Theme

struggling to bridge the inter-generation gap
Abstinence as the main content of sexual upbringing

\section{Control, monitoring and restriction}

The main approachof most parents involved in the sexuality upbringing of boys was to take supervisory action and attempt to limit their access to sexual information.Parents perform controlling functions through tangible and intangible monitoring ways in receiving sexual information, controlling and limiting communication and monitoring interaction with peers of the same and opposite sex.Acknowledging the inefficiencv of adnlescent restriction thev consider the communication technology in the sexuality Loading [MathJax]/jax/output/CommonHTML/fonts/TeX/fontdata.js 
upbringing of boys as an important negative factor as it makesmonitoring and restriction of adolescents difficult for parents; hence, they do not consider sexuality upbringing limited to parents.

"In my opinion, there is no to close the adolescents' access to sexual information, and it is almost impossible. They can do everything by their cellphones, and read and see sexual matters in most precise details with photos and videos" (p. 4, 40-year-old father).

However, other parents believe that extreme restraint leads to adolescents' secrecy and cause irreparable harm:

"Any extreme pressure and limitation will undoubtedly have a reverse effect and makes the explosion of this need in a much worse and dangerous way. I think that we should have the internet, satellite, mobile, and even girl-friend, but with repeated reminders of dangers and remote control". (p.4,40-year-old)

Parents' reaction to their children's sexual questions was silence and duplication by referring teenager to another parent or giving unrealistic and childish responses or delaying until adulthood.

\section{Abstinence as the main content of sexual upbringing}

Most parents tried to deviate their children's thoughts from sexual issues by forcing them to focus on lessons or sports activities in an effort to inhibit adolescents' sexual desire.According to parents' opinion, sexual relationship before marriage was sexual deviationand promiscuity,thus they should learn how to abstain until marriage.The educational process of most parents was to inhibit the instinctive desire inadolescents, warning, teaching, and advising on the negative consequences of sexual conduct in adolescence.Parents warn about adolescent false attachment, the loss of focus on their lessons and their future, negative psychological effects of watching porn movies, and the pathogenicity ofmasturbation.Parents were pessimistic about friendship with the opposite sex, and they considered it as an introduction to illegitimate sex. This belief could be found in all of their sentences:

"It's not just a matter of friendship. I feel they are seeking the real sex and they easily sleep with their girlfriends". (p4,40-year-old)

"I completely disagree with relationships between girls and boys at this age; and my son knows if he makes mistakes, how I can react to him. When we watch TV series or movies about problems of street friendship for families. I emphasize it in front of my children to be aware and if they have untimely sexual matters, it will end in the misdirection and even scandal". (p3, 43-year-oldfather)

Religious parents also resorted to religion in their efforts to inhibit adolescent sexuality orientations and considered virtue as an important factor in adolescent self-control.

"Nothing like prayer can prevent deviations of youth. I try to encourage children to pray even by force (he laughs). I do not let them cut off their relationships with Quran and mosque. Children are always present in the relininuc ceremnniec nf nur relatives (n2 42 -vear-nldfather).

Loading [MathJax]/jax/output/CommonHTML/fonts/TeX/fontdata.js

Page 6/14 


\section{Endeavoring for peace and tolerance}

In contrast, some parents believed that empathy, positive and hopefulimpressions of physical/emotional changes of puberty, as well as the avoidance of violent behavior could effectively control the sexual behavior in adolescents. In their view, the empathy with adolescents built mutual trust with parents as an important factor in adolescent adherence to family values.

"His mother saw in his cellphone that he had exchanged sexual messages and pictures with a girl. We logically tried to explain to him that it was very wrong. That's it! I told her that it's too dangerous and can go both of you to hell. I talked to her more intimately. I saw that he was so scared. I said: "no problem! I understand. These tendencies are natural. I was the same as you when I was young". He apologized and it ended". (p4,40-year-old)

\section{Criticizing the pathologic attitude to sexual instinct}

The knowledgeable parents had positive attitudes towards sexuality and tried to pass this era of their son's development and growth by desensitizing sexual issues and considering their behavioral changes asnormal matters.Criticizing the negative attitude towards sexuality in the Iranian culture and referring to its destructive effects on sexual relations in the future of marriage the parents believed that the sexuality should not be neglected or suppressed, but it should be considered with a more open mind and we should try to positively lead it instead of radical restrictions.

"Sexual issues are not all negative, they should not be considered as taboos. It makes the issue more complicated. In Iran, sexual matters have been negatively considered. The current culture makes our kids non-informed and non-properly trained, and finally, they do not fully enjoy sexual matters due to their fear. We can emphasize positive, enjoyable and vital aspects of sexuality". (p4, 40-year-old).

Some parents know about the importance of providing an appropriate sexual education context as its basic prerequisite.For example, they cangive the teenager the courage to express sexual issues by creating intimacy with them or talk about privacy in jokes in order to create sexual communication.

"I tell him: naughty! you have spent an hour in front of the mirror, do have you a date today? (with a smile). It gives him courage and makes intimacy little by little. So, if he had a relationship with someone or had any problem, I know what to do, and he can tell me about it".(p2,38-year-old father)

Some parents believed that the desensitization of communication with the opposite sex would lead to the normal assumption of sexual tendencies which unlike its perception could result in delayed sexual experience in adolescents. Therefore, they allowed experiencing first communication with the opposite sex under parental supervision.

"I do not want the communication with the opposite sex to be a taboo and inaccessible and special issue for him. That's too bad. He thinks that it definitely has special things that should be explored on his own. I 
think that assuming it as a normal issue can help him to continue the same for more years, and then he can directly experience it". (p5, 42-year-old mother)

"Resistance and complaining about this issue lead to no result. Therefore, if he went to military service and university, he will be "Superficial" and immediately falls in love if he faces a girl's coquetry. If I allow my son to experience everything at home, it will have better result and my concern will be also less" ( $\mathrm{p} 2$, 38-year-oldfather)

Referring to the impossibility of a complete restriction of adolescents, especially boys, parents believed that instead of limiting interactions that were ineffective or low-effective in today's society, they should empower teenagers to reduce their vulnerability. They considered it as a kind of child immunization to protect against the dangers of society.

"I like to make him a bit free, that is, if he probably gains some dangerous or inappropriate things, I will consider it as a vaccine that can immunize him against bigger storms". (53-year-old father)

\section{Hope for social learning}

It is worth mentioning that even some educated parents, who have allocated sex education to natural movement, hope for social learning.

"I have tried to let him gain this information naturally, through his dispersed findings. I gave him freedom, but I have also monitored him. I was probably wrong, but as I learned it (sexuality) from nobody and gradually learned it from the social environment, especially university, I think this option is appropriate. It means giving freedom to children by the natural and controlled way, especially nowadays that there are social networks". (53-year-old father)

In their opinion, in families with ethical values and controlled adolescent relationships, it is not a major concern even if they do not directly talk about sexual education:

"The reason why I left my son's education in this field is that, given the safe atmosphere in the family, I have the peace of mind that he will safely pass this crisis since it had happened to me and made no problem for me. I partially trust him as I knew his personality. His connection with the outside is limited to a few friends, so it is not a concern for me or at least I am optimistic"(53-year-oldfather)

Emphasizing on the educational effect of parents' behavior on adolescents' behavior, some parents tried to be a model for their adolescents, especially in social interactions with the opposite sex:

"I want my son to imitate me as much as possible not anyone else. I have seen the impact of this behavior on my son. If I model myself, I would better affect him". (p4,40-year-old)

Parents preferred metaphorical and indirect education to direct discussion of sexual issues.For instance, teaching to respect individual privacy as a metaphor for the existence of private relationships. From their Loading [MathJax]/jax/output/CommonHTML/fonts/TeX/fontdata.js ity. 
"We taught him how to respect people's privacy from childhood, that women and men may have privacy issues. In contrast, when we knocked at the door of his room, it respects his dignity and he feels safe if he has something in his privacy". (p5, 42-year-old mother)

None of the parents referred to books or any kind of education about the sexual upbringing of their children. This issue reflects the neglect of parent education in this regard.

"Unfortunately, because we are not trained in this field, I do not know what the ideal way is. Should the family play a $100 \%$ role? Is it 50-50 between school and family? Is it possible by referring a teenager to read a book? I have not seen any book in this field. I am almost illiterate in this regard. I have a big question in my mind that what the ideal approach is? Perhaps, there is no a single ideal approach".(p5, 53-year-oldfather)

\section{Discussion}

The emerged themes from parents' experiences and perceptions of sexuality upbringing of adolescent boys indicated the parents' confusion, uncertainty and ineffectiveness in confronting their sexual education. The underlying foundation of all approaches ultimately ends in the establishment of abstinence as the parents' red line. Parents struggle with their beliefs, norms of society and their sons' expectations and beliefs. This confrontation results in a set of conservative policies by parents, so that they can maintain their power and control performance, decrease the intergeneration gap and utilize a kind of peaceful upbringing to decrease tensions and challenges of sexual upbringing. They use an intermediate behavior by relying on their teachings influenced by the huge intergeneration difference in consideration of the sexual dimension.

A recent study in Iran indicated that parents' beliefs about sexuality upbringing of children are fragilely oscillating. They are not aware of what sexuality upbringing is and how sexuality ethics is taught. Their approach to sexuality upbringing is a contingency approach, in other words, just when they have a problem, they think about the solution. Until a problem has arisen, they do not explicitly talk about sexual issues, and even sometimes consider it as the reason for attracting adolescents to sexual behavior [17].

Studies indicate that the content of parenting messages is more focused on the self-control, while peer and media messages are significantly about the positivity of sex $[8,18]$. Adolescents talk to their peers about the details of sexual issues [19]. Parents seem to monitor teenagers' access to sexual information resources and limit their interaction with peers.

As mentioned above, parents take efforts to suppress their sons' sexual desire and behavior by resort to ways to strengthen religious beliefs, counseling and warning about sexual behavior. Similarly, in other studies, Christian parents have used church attendance or reading the Bible as ways for their children's sexual upbringing instead of talking to them directly. Religious parents talk less about sexual issues or methods of contraception with their adolescents, and they first emphasize religious values and self- 
According to a study on the sexuality upbringing style in the Iranian families, they had more attention, even insufficient, to sexual orientation and morality, but the least attention to the sexual awareness [22]. In the present study, none of the parents talked about the protected sex in their sons' sexuality upbringing. Despite the fact that parents implicitly had uncertainties about their adolescents' immunity and concern for their sexuality, they limited the sexuality upbringing to self-control, and it is noteworthy that none of the parents referred to teaching skills which enabled teenagers to self-control. Even though some of them referred to the inefficiency of restriction, they considered the control, not the empowerment, as the appropriate solution. The life skills approach is considered as the skill-based sexual health education by such international institutions such as the WHO, UNESCO, UNFPA in the adolescent sexuality upbringing.

One of the significant findings of the present study was the positive attitude of some parents to the sexuality and attempt to desensitize sexual taboos that formed the basis of their attitudes towards the sons' sexuality upbringing. This finding was inconsistent with previous studies on girls in Iran over the past few decades. Iranian adolescent girls criticized the negative attitude of adults towards the sexuality and their resort to frightening and blackmail of sex consequences [23]. In fact, socio-cultural changes of Iran in recent decades have changed the parents' attitude to the necessity and type of sexuality upbringing for children. Nevertheless, a recent study in Iran has indicated that $82 \%$ of parents still have not talked about sexual affairs with their children [24]. This is mainly due to the lack of parents' training on how to train important sexual issues. Studies have indicated that there is a chain relationship between parent's attitudes and children attitudes towards the sexuality and parent-child dialogue about sexuality with adolescents' sexual behavior [25].

There are several theories indicating that effective sexuality upbringing should increase sexual health within individual values, ethical beliefs, and ethnic and religious backgrounds [26]. Sexuality upbringing will be successful if the cultural context is not ignored $[27,28]$. Etzioni writes that what is important in sexual education is sex education and morality. The most important point is that this sexual morality is based on family values. Families have both rights and responsibilities in sexuality upbringing [29, 30]. Parents are the most natural sex educators for teenagers. They have the best position to support adolescents to face their ambiguity of sexual life [31]. According to our findings, Iranian parents consider themselves more entitled than responsible.

In our study, some parents expressed their optimism about the effectiveness of their monitoring and positive impact of the family's upbringing atmosphere. They considered the unlikelihood of their adolescents` engagement in sexual function, thus they were sure that, firstly, there was little need to directly discuss sexual issues with their teenagers, and secondly, there was no serious risk threatening their adolescent. Eisenberg indicated that parents who were sure that their children are engaged in romantic relationships, were more likely to talk to their teenagers about sexuality [32]. Previous studies reported the impact of parents monitoring on adolescents' sexual behaviors [33].

Surprisingly, Comparison of findings of this study with previous studies indicated that the middleclacclelite narentc in Iran have relativelv cimilar heliefc about the necessity of abstinence from premarital Loading [MathJax]/jax/output/CommonHTML/fonts/TeX/fontdata.js

Page $10 / 14$ 
sex for both their sons and daughters [6]. Similarly, challenges that Iranian parents face in sexual education are the same for both male and female adolescents [34, 35]. A recent study in Iran showed that parents of adolescent boys have unmet educational needs in sexuality education. They believed that both teachers and parents should be trained for sexuality education [35].

The present study was among the first studies address the sexuality education of adolescent boys in Iran. However, it has some limitations; the participants were limited to middle/high class families that were not necessarily representatives of the population. Future studies can enrich data by explaining sons' experiences of sexuality education by their parents.

\section{Conclusions}

Findings imply that Iranian parents are facing uncertainty regarding sexual education for their adolescent boys. All approaches finally resulted in abstinence. Abstinence is the central concept in their sexuality upbringing style for their sons. so, sexual relationship is a red line issue in sexuality upbringing. Their conservative peaceful approach tries to reduce the intergeneration gap. These findings provide evidence for culturally sensitive approaches to address educational interventions for parents to improve their skills and knowledge about sexuality education for their children.

\section{Abbreviations}

WHO

world health organization

UNFPA

united nations population fund

UNESCO

united nations educational, scientific and cultural organization

\section{Declarations}

\section{Ethics approval and consent to participate}

The study protocol was approved at the Ethics Committee of Ahvaz Jundishapur University of Medical Sciences (Ethic code: IR.AJUMS.REC.1395.187).

Consent for publication: Not applicable

\section{Availability of data and materials:}

The datasets used and/or analyzed during the current study are available from the corresponding author on reasonable request. 
The authors declare that they have no competing interests.

\section{Funding:}

This article is part of the M.S. thesis in reproductive health belonged to the first author. The research deputy of Ahvaz Jundishapur University of Medical Sciences financially supported this study (Grant code: RHPRC-9502).

\section{Authors' contributions:}

ZR collected and analyzed data, and wrote the initial draft of manuscript; MJ supervised the study and participated in conceptualization and designing the study, analyzing and interpretation of data, and critically revised the manuscript; SS participated in analyzing and interpretation of data and writing the initial draft. All authors read and approved the final manuscript.

\section{Acknowledgments}

The authors are deeply grateful to all parents participating in the research as well as the research deputy of Ahvaz Jundishapur University of Medical Sciences for their financial support

\section{Authors details:}

1-School of Nursing and midwifery, Ahvaz Jundishapur University of Medical Sciences, Ahvaz, Iran

2- Reproductive Health Promotion Research Center, Ahvaz Jundishapur University of Medical Sciences, Ahvaz, Iran

3- Department of Reproductive Health and midwifery, Faculty of Medical Sciences, Tarbiat Modares University, Tehran, Iran

\section{References}

1. Comprehensive Sexuality Education: Advancing Human Rights, Gender Equality and Improved Sexual and Reproductive Health. UNFPA 2010

2. Homma Y, Wang N, Saewyc E, Kishor N. The Relationship between Sexual Abuse and Risky Sexual Behavior among Adolescent Boys: A Meta-Analysis. J Adolesc Health. 2012 Jul; 51(1): 18-24, doi: 10.1016/j.jadohealth.2011.12.032

3. Widman L, Choukas-Bradley S, Noar SM, Nesi J, Garrett K. Parent-Adolescent Sexual Communication and Adolescent Safer Sex Behavior: A Meta-Analysis. JAMA Pediatr. 2016;170(1):52-61. doi:10.1001/jamapediatrics.2015.2731

4. Fonner VA, Armstrong KS, Kennedy CE, O'Reilly KR, Sweat MD. School Based Sex Education and HIV Prevention in Low- and Middle-Income Countries: A Systematic Review and Meta-Analysis. (2014), 
5. Sridawrang C, Pfeil M, Krozier K. why Thi parents do not discuss sex with their children: a qualitative study. 2010. Nurs health science, 12(4)437-443

6. latifnejad Roudsari R, Javadnoori M, Hasanpour M, Hazavehei S M M, Taghipour A. Socio-cultural challenges to sexual health education for female adolescents in Iran. Intr J Reprod BioMed 2013;11(2):101-10.

7. UNESCO. International technical guidance on sexuality education. An evidence-informed approach. Revised edition 2018, available at: https://unesdoc.unesco.org/ark:/48223/pf0000260770

8. Epstein M, Ward LM "Always Use Protection": Communication Boys Receive about Sex from Parents, Peers, and the Media. J Youth Adolesc, 2008. 37:113-126. Doi: 10.1007/s10964-007-9187-1

9. Lindberg L D, sonfield A, Gemmill A. Reassessing Adolescent Male Sexual and Reproductive Health in the United States: Research and Recommendations. Am J mens health. 2008;2(1):40-56. doi:1177/1557988307309460

10. Malek A, Abbasi Shokoohi H, Bina M, Shafiee-Kandjani A. A study on source of sexual knowledge acquisition among high school student in Northwest Iran. Arch Iran Med. 2010;13(6):537-42. https://www.ncbi.nlm.nih.gov/pubmed/21039011

11. Garmaroudi Gh.R, Makarem J, Alavi S.Sh, Abbasi Z. health related risk behavior among high school students in Tehran, Iran. Payesh, 2010: 9(1), 13-19 (full text in Persian)

12. Bahrami N, Simbar M, Vedadhir A, et al. "Male Iranian adolescent's reasons for having an othergender friend" International Journal of Adolescent Medicine and Health, 29.3 (2016): -. Retrieved 10 Apr. 2019, from doi:10.1515/ijamh-2015-0086

13. Leylabadlo HE, Baghi HB, Fallahi L, Kafil HS. From sharing needles to unprotected sex: a new wave of HIV infections in Iran? Lancet HIV. 2016; 3: e461-e462

14. Ott M A. Examining the Development and Sexual Behavior of Adolescent Males Published in final edited form. J Adolesc Health. 2010 April; 46(4): S3-11. doi: 10.1016/j.jadohealth.2010.01.017

15. Graneheim UH, Lundman B. Qualitative content analysis in nursing research: concepts, procedures and measures to achieve trustworthiness. Nurse Educ Today. 2004 Feb;24(2):105-12. DOI: 10.1016/j.nedt.2003.10.001

16. Shenton AK. Strategies for ensuring trustworthiness in qualitative Research Projects. Education for Information 2004; 22(2): 63-75. DOI: 10.3233/EFI-2004-22201

17. Bolandhematan K. Representation of how parents lived experience of sexual ethics education to their children, family counseling and psychotherapy Quarterly, Issue 4, winter 2015. (In Persian).

18. Bleakley A, Hennessy M, Fishbein M, Coles H C, Jordan A. How source of sexual information relate to adolescents' beliefs about sex. Am J Health Behavior, 2009; 33, 37-48

19. Heisler, J. M. Family communication about sex: Parents and college-aged offspring recall discussion topics, satisfaction, and parental involvement. Journal of Family Communication, (2005). 5, 295-312.

20. Regnerus M. D. Talking about sex: Religion and patterns of parent-child communication about sex and contraception. The Sociological Quarterly, (2005).46, 79-105.

Loading [MathJax]/jax/output/CommonHTML/fonts/TeX/fontdata.js

Page 13/14 
21. Kim JL\& Ward, M. Silence speaks volumes: Parental Sexual Communication Among Asian American Emerging Adults. Journal of Adolescent Research. (2007), 22, 3.

22. Sobhaninejad M, Homaee R, Elieen H. Analysis of the Style of Sex Education in Families of Tehran. Counseling Research and Developments. 2007; 6(21), 27-48. (In Persian).

23. Javadnoori M, Latifnejad Roudsari R, Hassanpour M, et al. Female adolescents' experiences and perceptions regarding sexual health education in Iranian schools: A qualitative content analysis. Iran J Nurs Midwifery Res. 2012 Nov-Dec; 17(7): 539-546.

24. Ganji J, Emamian MH, Maasoumi R, Keramat A, Merghati Khoei E. Sexual Health Education at Home: Attitude and Practice of Iranian Parents. Iran J Public Health. 2018;47(1):146-147.

25. Schouten BC, van den Putte B, Pasmans M, Meeuwesen L. Parent-adolescent communication about sexuality: The role of adolescents' beliefs, subjective norm and perceived behavioral control. Patient education and counseling. 2007; 66(1):75-83.

26. McKay A. Accommodating Ideological Pluralism in Sexuality Education; Journal of Moral Education (1997). 26, 3: $285-300$

27. Mkumbo K and Ingham R. What Tanzanian parents want (and do not want) Covered in school-based sex and relationships education; Sex Education (2010). 10(1): 67- 78

28. Rawson $\mathrm{H}$ and Liamputtong P. Culture and sex education: the acquisition of Sexual knowledge for a group of Vietnamese Australian young women; Ethnicity \& Health (2010). 15, 4: 343- 364

29. Etzioni A. Sex Education Should Emphasize Values; in: Sex Education; Edited by: Tamara L. Roleff; Greenhaven Press, Inc. San Diego, California, 1999.

30. McCarthy, John F. Sex Education Should Be Taught Primarily by Parents; in: Sex Education; edited by: Tamara L. Roleff; Green haven Press, Inc. San Diego, California; 1999.

31. Halstead M J. Muslims and sex education; Journal of moral education, (1997). 26, 3:317-330

32. Eisenberg M E, Bearinger L H, Resnik M D. Parents' communication with adolescents about sexual behavior: a missed opportunity for prevention? J youth Adolescence. 35: 893-902 (2006).

33. Dittus P J, Michael S L, BecasenJ S, Gloppen K M, McCarthy K, Guilamo-Ramos V. Parental Monitoring and Its Associations With Adolescent Sexual Risk Behavior: A Meta-analysis. Pediatrics Dec 2015, 136 (6) e1587-e1599; DOI: 10.1542/peds.2015-0305

34. Rouhparvar Z, shahali S, Jvadnoori M. parentsperceptionsofchal $\leq n \geq$ sofma $\leq$ ado $\leq$ scents sexual education: A qualitative study. J Qual res health sci 2019; 8[3]: 260-70. [In Persian]

35. Babayanzad Ahari, S, Behboodi Moghadam, Z, Azin, S.A. et al.Concerns and educational needs of Iranian parents regarding the sexual health of their male adolescents: a qualitative study. Reprod Health17,24 (2020). https://doi.org/10.1186/s12978-020-0883-6 